\title{
A Commercial Conducting Polymer as Both Binder and Conductive Additive for Silicon Nanoparticle- Based Lithium-Ion Battery Negative Electrodes
}

Thomas M Higgins, ${ }^{1,2 *}$ Sang-Hoon Park,,${ }^{1,3^{*}}$ Paul J King,,${ }^{1,4}$ Chuanfang (John) Zhang, ${ }^{1,3}$ Niall McEvoy, ${ }^{1,3}$ Nina C. Berner, ${ }^{1,3}$ Dermot Daly, ${ }^{1,2}$, Aleksey Shmeliov, ${ }^{1,3}$ Umar Khan, ${ }^{1,2}$ Georg Duesberg, ${ }^{1,3}$ Valeria Nicolosi, ${ }^{1,3 * *}$ Jonathan N Coleman, ${ }^{1,2 * *}$

${ }^{1}$ Centre for Research on Adaptive Nanostructures and Nanodevices (CRANN) \& Advanced Materials Bio-Engineering Research Centre (AMBER), Trinity College Dublin, Dublin 2, Ireland.

${ }^{2}$ School of Physics, Trinity College Dublin, Dublin 2, Ireland

${ }^{3}$ School of Chemistry, Trinity College Dublin, Dublin 2, Ireland

${ }^{4}$ Bell Labs Ireland, Blanchardstown Business \& Technology Park, Snugborough Rd, Dublin 15 , Ireland

*These authors contributed equally

**nicolov@tcd.ie,colemaj@tcd.ie

KEYWORDS: anode, battery, binder, conducting polymer, conducting additive, negative electrode, PEDOT:PSS, silicon. 


\section{ABSTRACT}

This work describes silicon nanoparticle-based lithium-ion battery negative electrodes where multiple non-active electrode additives (usually carbon black and an inert polymer binder) are replaced with a single conductive binder; in this case the conducting polymer PEDOT:PSS. While enabling the production of well-mixed slurry-cast electrodes with high silicon content (up to $95 \mathrm{wt} \%$ ), this combination eliminates the well-known occurrence of capacity losses due to physical separation of the silicon and traditional inorganic conductive additives during repeated lithiation/delithiation processes. Using an in situ secondary doping treatment of the PEDOT:PSS with small quantities of formic acid, electrodes containing $80 \mathrm{wt} \%$ SiNPs can be prepared with electrical conductivity as high as $4.2 \mathrm{~S} / \mathrm{cm}$. Even at the relatively high mass loading of $1 \mathrm{mg} / \mathrm{cm}^{2}$, this system demonstrated a first cycle lithiation capacity of $3685 \mathrm{mAh} / \mathrm{g}$ (based on the SiNP mass) and a first cycle efficiency of $\sim 78 \%$. After 100 repeated cycles at 1 $\mathrm{A} / \mathrm{g}$ this electrode was still able to store an impressive $1950 \mathrm{mAh} / \mathrm{g}$ normalised to Si mass ( $75 \%$ capacity retention), corresponding to $1542 \mathrm{mAh} / \mathrm{g}$ when the capacity is normalized by the total electrode mass. At the maximum electrode thickness studied $\left(\sim 1.5 \mathrm{mg} / \mathrm{cm}^{2}\right)$ a high areal capacity of $3 \mathrm{mAh} / \mathrm{cm}^{2}$ was achieved. Importantly, these electrodes are based on commercially available components and are produced by the standard slurry coating methods required for large-scale electrode production. Hence, the results presented here are highly relevant for the realisation of commercial $\mathrm{LiB}$ negative electrodes that surpass the performance of current graphite-based negative electrode systems. 
The development of Li-ion batteries ( $\mathrm{LiBs}$ ) with improved performance is necessary to fulfil the requirements of future consumer electronics, electric vehicles, and renewable energy storage grids. ${ }^{1,2}$ At present, graphite is the mainstay negative electrode material for commercial $\mathrm{LiBs}$, which can theoretically provide a maximum specific capacity of $C_{s p}=372 \mathrm{mAh} / \mathrm{g}$. While research has pushed into negative electrodes based on graphene, ${ }^{3-6}$ a number of other negative electrode materials potentially offer much higher capacity. Silicon, in particular, has an extremely large theoretical specific lithiation capacity of $C_{s p}=3579 \mathrm{mAh} / \mathrm{g}$ (corresponding to its maximum metastable alloying composition of $\mathrm{Li}_{15} \mathrm{Si}_{4}$ at ambient temperatures), a very low lithiation/delithiation potential, and is both safe and abundant. As such, the use of silicon is extremely compelling for the development of $\mathrm{LiB}$ negative electrodes with performance that exceeds the current state-of-the-art graphite-based negative electrodes.

While silicon-based electrodes often possess exciting capacities during the initial cycles, it is commonly found that these high values cannot be maintained for the hundreds of cycles that are needed to make silicon a viable replacement for graphite. Ultimately, this is due to the large volume change that is associated with such a substantial uptake of lithium into the silicon structure $\left(\sim 300 \%\right.$ for $\left.\mathrm{Li}_{15} \mathrm{Si}_{4}, \operatorname{Ref}^{7}\right)$. As a consequence, electrode failure occurs due to various modes such as pulverization of the active material, loss of electrical pathways following electrode structural rearrangement, ${ }^{8-10}$ continuous instability of the solid/electrolyte interfacial (SEI) layer, ${ }^{11,12}$ or some combination of these. It is clearly necessary that an electrode formulation be developed which can overcome all of these issues.

The problem of pulverisation of the silicon active material has largely been resolved through the use of various nanostructured forms of silicon (e.g. nanoparticles, nanowires, and other nanoporous structures) $)^{13-17}$ which can undergo deep lithiation and delithiation processes with excellent reversibility. ${ }^{14,17}$ Consequently, attention is increasingly moving beyond the active component of the electrode and towards optimising the overall electrode structure.

In this arena, it is becoming clear that optimisation of the binder material is pivotal for the realisation of high capacity-retention silicon-based negative electrodes. ${ }^{18,}{ }^{19}$ In general, binders that are used in battery electrodes must; (1) be capable of forming a homogenous mixture with the active component and the conductive additive; (2) provide suitable binding interactions with both the active material and conductive additive leading to stable electrical pathways to 
the current collector, and (3) be electrochemically stable in the required electrochemical potential window. However, for electrodes that undergo large volume changes there are a number of additional binder requirements; this component must (4) possess mechanical properties that can reversibly buffer the requisite volume changes of the active material, (5) undergo minimal swelling in the electrolyte, (6) enable the creation of a thin and stable SEI layer, and (7) maintain adhesion between the overall composite structure and the underlying current collector. Further to these technical requirements, to be economically viable a candidate binder must also be (8) easy to produce in large quantities, (9) inexpensive, and (10) compatible with standard electrode manufacturing techniques (i.e. slurry casting).

Clearly, arriving at a material that meets all of these requirements is a significant challenge. This is reflected by the wide range of polymeric binders that are presently being explored, including synthetic polymers such as polyamide imide, ${ }^{14}$ lithium-exchanged nafion, ${ }^{20}$ polyacrylic acid (PAA), ${ }^{21}$ and polyimide, ${ }^{22}$ biopolymers such as carboxymethyl cellulose $(\mathrm{CMC}),{ }^{23}$ alginate, ${ }^{24}$ and cyclodextrin, ${ }^{25}$ as well as combinations of these such CMC/styrene butadiene rubber $^{26}$ or PAA/polyvinyl alcohol. ${ }^{27}$ Among these studies, some authors have focussed on imparting elastomeric properties to the electrode matrix to improve the cycling performance. ${ }^{18,19,23,26}$ In others, more emphasis has been given to optimising the surface interaction between silicon and the binder as to avoid irreversible rearrangement of the electrode structure. ${ }^{28}$ However, success ultimately depends on arriving at a binder system that satisfies all of the above-mentioned criteria simultaneously.

Meeting this challenge will be far more likely if the number of binder requirements can be reduced. We believe that the most compelling strategy to do so is through the use a conducting polymer in place of the more commonly explored insulating varieties such as those mentioned above. In particular, the use of a conducting polymer binder immediately eliminates the tenuous interface between silicon and conventional inorganic conductive additives such as carbon black, carbon nanotubes or graphene. This presents an opportunity to either suppress, or ideally, completely avoid capacity losses due to the physical separation of the active material and the conductive phase. Furthermore, the use of a conductive binder in place of a traditional binder/conductive additive combination means a reduced parameter space and an opportunity to minimize the presence of non-active components within the electrode formulation. ${ }^{29,30}$ Clearly, this will favour the overall gravimetric capacity of an electrode, which underlies the use of silicon within $\mathrm{LiB}$ negative electrodes in the first place. 
Although there has been much interest in the use of conductive polymers within LiBs generally (see the recent review by Sendodu et al. ${ }^{31}$ ), relatively little work has been done to explore the combination of conducting polymers with silicon for the creation of commercially relevant LiB negative electrodes. A number of prior studies have combined silicon with polyaniline, ${ }^{32}$ pyrene-modified methacrylate, ${ }^{33}$ 3,6-poly(phenanthrenequinone), ${ }^{29}$ various polyfluorenebased polymers, ${ }^{30,} 34-37$ and poly(3,4-ethylenedioxythiophene)/poly(styrene-4-sulfonate) (PEDOT:PSS). ${ }^{38-40}$ While these works call attention to the efficacy of using conducting polymers to improve silicon-based negative electrodes, many of these polymer systems require complicated in-house synthesis for which commercial viability of their production is unknown, ${ }^{29}, 30,33-37$ use electrode production methods other than industry favoured slurry coating, ${ }^{38-40}$ or require additional binders, ${ }^{37,40}$ conductive additives, ${ }^{37,39,40}$ or non-aqueous solvents for their production. ${ }^{29,33-36}$

On the other hand, PEDOT:PSS is commercially available at relatively low cost and has been demonstrated to be a versatile host for a wide range of inorganic nanomaterial fillers for various electrochemical energy storage/conversion technologies. ${ }^{41}$ It possesses excellent electrochemical stability (even when repeatedly n-doped), ${ }^{42,43}$ is mechanically robust, ${ }^{44}$ and through the use of simple secondary doping treatments it can possess impressive electrical conductivity. ${ }^{45}$ In addition, due to its solubility in water PEDOT:PSS is easily processable using conventional techniques; it can be used to form homogenous slurries with the active material without the need for hazardous/expensive solvents. ${ }^{42}$ Due to these numerous advantages, in this work we investigate the performance of silicon nanoparticle (SiNP) based LiB electrodes in combination with PEDOT:PSS as a commercially relevant, highperformance conductive binder. We find very high gravimetric capacities coupled with excellent stability and state-of-the-art rate behaviour. 


\section{RESULTS AND DISCUSSION}

General Morphological Characterisation: We have studied the properties of a LiB negative electrode material featuring PEDOT:PSS as a conductive binder in combination with SiNPs as the active component. Transmission electron microscopy (TEM) and X-ray photoelectron spectroscopy (XPS) were performed to examine the nature of the silicon nanoparticles. This data shows the SiNPs to be crystalline with a mean diameter of $\sim 60 \mathrm{~nm}$ and a surface oxide coating of thickness 2-5 nm (see Supporting Information, Figure S1 \& S2). Throughout this study we make comparisons with a relatively successful ${ }^{21}$ though conventional SiNPcontaining LiB negative electrode system consisting of $80 \mathrm{wt} \%$ SiNPs with $10 \mathrm{wt} \%$ carbon black (CB) and $10 \mathrm{wt} \% \mathrm{Li}$-PAA polymer binder. The experimental and conventional electrode systems are presented schematically in Figure 1a and b, respectively. For both electrode types the composites were prepared by hand-mixing the relevant components for 30 minutes using a ceramic mortar and pestle. The resulting slurries were then converted into films on $\mathrm{Cu}$ foil substrates by doctor blading. Figure 1c and 1d presents photographs of the two components for the experimental system and a resulting slurry cast film. For the PEDOT:PSS containing samples, in most cases a controlled quantity of formic acid (FA) was added to the slurry during mixing. The FA acts as a 'secondary dopant' for PEDOT:PSS to dramatically improve its electrical properties. ${ }^{45,46}$ The effect of the FA loading within the slurry on electrode performance is discussed in the sections following.

Typical scanning electron micrograph (SEM) images of the experimental (PEDOT:PSS/SiNP) and conventional (CB/PAA/SiNP) systems are shown in Figure 1e and f, respectively. These images indicate that the SiNPs that have been used are approximately spherical with diameters generally less than $200 \mathrm{~nm}$, appearing consistent with the mean diameter of $60 \mathrm{~nm}$ indicated by the supplier. In previous studies of Si-based negative electrodes it has been found that silicon-lithium alloying leads to mechanical fracture of the active material, particularly at high Li capacities. ${ }^{17}$ As such, we have chosen small diameter SiNPs for this study to avoid this undesirable process. In addition, this active material was used as opposed to other nanostructured forms of silicon (such as nanowires ${ }^{15}$ or core-shell structures ${ }^{47}$ ) as they are able to be produced relatively cheaply and in large quantities. For these reasons Si-based negative electrodes featuring SiNPs are likely to be of greater commercial interest than more complicated alternatives requiring multi-step processing or high temperatures during synthesis. 
To maximize the performance of any composite electrode system it is important that the components be uniformly mixed. We have assessed this aspect of these PEDOT:PSS/SiNP composites using SEM with electron back scattering (EsB) as well as energy dispersive X-ray (EDX) spectroscopic mapping. The Supporting Information presents wide-view SEM images of the PEDOT:PSS/SiNP and CB/LiPAA/SiNP systems both prepared using $80 \mathrm{wt} \% \mathrm{SiNPs}$ (Figure S3a and S3b, respectively), as well as EsB maps of the corresponding areas (Figure S3c \& S3d, respectively). These indicate excellent mixing for the conductive binder-containing system which is corroborated by EDX elemental mapping performed on another region of the same sample (Figure 1g). Here we have used the EDX signal due to sulphur and silicon to indicate the presence of the conducting polymer and nanoparticle components (Figure $1 \mathrm{~h} \& 1 \mathrm{i}$, respectively); PEDOT and PSS both possess sulphur atoms as shown by the chemical formula in the Figure $1 \mathrm{~g}$ inset. This suggests that the two constituents are homogeneously distributed down to length scales approaching the dimensions of the SiNPs, and shows the mixing of these materials to be extremely effective.

On the other hand, SEM and EsB images of the conventional system reveals micron-scale nonuniformity, with regions of predominately $\mathrm{CB}$ interspersed within regions of predominately SiNPs. Figure $1 \mathrm{j}$ presents a closer view of the film with the corresponding EsB map (Figure $1 \mathrm{k}$ ) indicating considerable segregation of the electrode components. This is not ideal as it means that a proportion of the available SiNPs will not be in close proximity to the conductive additive (and possibly also the polymer binder) and likely produce sub-optimal electrode performance, particularly during the repeated cycling of a large volume change active material.

Effect of Formic Acid Secondary Dopant on composite properties: We then examined the influence of the FA secondary doping treatment on the electrical and lithium storage properties of these composites. It is known that a range of polar and protic molecules can act as secondary dopants for PEDOT:PSS, inducing large increases in polymer conductivity. ${ }^{45,46,48-50}$ It has been suggested that secondary doping treatments impart morphological changes to the PEDOT conjugated polymer chains that are retained even after the dopant has been removed (through heating of the treated films). ${ }^{51}$

FA was chosen as the secondary dopant for this study as this molecule has previously been shown to be very effective for improving the properties of PEDOT:PSS transparent conductors and supercapacitor electrodes (films with thickness less than $\sim 1$ micron). ${ }^{45,46}$ Unlike these 
studies where the PEDOT:PSS has been exposed to the FA as a post-processing step (by immersion in a bath of the secondary dopant for a few seconds), ${ }^{45,} 46$ here we have added a quantity of the FA directly into the mortar while mixing the composite slurries (i.e. in-situ secondary doping). This method was used as it was found that FA secondary doping by immersion caused corrosion of the $\mathrm{Cu}$ foil current collector and also caused the composite to delaminate from its substrate. By mixing the secondary dopant directly into the slurry both these problems were avoided while also simplifying the film production process. To our best knowledge this is the first report of a FA secondary doping treatment applied in situ to thick composite films. Table S1 in the Supporting Information gives details of the mass loading, thickness and density for the various samples of different compositions that were prepared.

To optimize the electrical conductivity increase arising due to this in-situ FA treatment we prepared a range of $20 \mathrm{wt} \%$ PEDOT:PSS/SiNP samples on glass slide substrates with FA loadings up to $20 \%$ (relative to the volume of the PEDOT:PSS solution). After oven drying of samples, four-wire IV measurements were then performed to determine the sheet resistance, $\mathrm{R}_{\mathrm{s}}$, of replicate samples following the creation of parallel electrical contacts on each film with conductive silver paint. The mean thickness, $t$, of each sample was also determined using contact profilometry, enabling calculation of the DC conductivity using $\sigma_{D C}=\left(R_{s} t\right)^{-1}$

Figure 2 a shows that a $100 \times$ increase in $\sigma_{D C}$ could be achieved by the addition of $10 \%$ FA to the composite, giving $\sigma_{D C}=4.2 \mathrm{~S} / \mathrm{cm}$ (the FA-free control had a $\sigma_{D C}=36 \mathrm{mS} / \mathrm{cm}$ ). The addition of FA in quantities beyond this value provided no additional improvements in conductivity. As such, 10\% FA was included during the preparation of all subsequent PEDOT:PSS/SiNP samples to maximize the film conductivity. We emphasize that even the composite samples prepared without any secondary-dopant had a DC conductivity that was $35 \times$ higher than the conventional Li-PAA/CB containing sample with the same 80 wt\% SiNP loading ( $\sigma_{D C}=1.1 \mathrm{mS} / \mathrm{cm}$, indicated by the red dotted line in Figure $2 \mathrm{a}$ ). It is worth noting that in addition to these impressive electrical properties, PEDOT:PSS is expected to be significantly more thermally stable compared to other conducting polymers. ${ }^{52}$

We then performed some initial electrochemical characterisation of these PEDOT:PSS/SiNP composites to assess the influence of this FA treatment on the resulting electrode performance. This was carried out in a half-cell configuration $v s$. lithium foil counter electrode inside a 2032type coin cell with $1 \mathrm{M} \mathrm{LiPF}_{6}$ in ethyl carbonate/dimethyl carbonate/fluoroethylene carbonate 
as the electrolyte $(3: 6: 1 \mathrm{v} / \mathrm{v})$. Galvanostatic charge and discharge measurements were performed using a $20 \mathrm{wt} \%$ PEDOT:PSS sample prepared without the addition of FA as well as identical samples prepared using 5\% and 10\% FA (again, relative to the volume of the PEDOT:PSS solution). The same measurements were also carried out on the conventional electrode system for comparison.

The first lithiation/delithiation cycle was carried out at a constant current density of $0.5 \mathrm{~A} / \mathrm{g}$ and the resulting voltage profiles are shown in Figure $2 b$. Here the specific lithiation/delithiation capacities of each electrode was calculated based on the mass of the active material only. All of these Si-based electrodes show a long plateau at $<0.15 \mathrm{~V}$ during the first lithiation which corresponds to the formation of a Li-Si alloy phase. However, there is a notable difference between $\mathrm{CB} / \mathrm{Li}-\mathrm{PAA} / \mathrm{SiNP}$ and PEDOT:PSS/SiNP electrodes in terms of voltage profiles during this initial lithiation stage; while the Li-PAA/CB/SiNP electrodes did not have a slope during initial lithiation, all of the PEDOT:PSS-containing electrodes show an obvious slope within certain voltage windows (1.0-0.015 V). This likely arises due to some irreversible reactions associated with PEDOT:PSS. The Supporting Information contains voltage profiles measured for a PEDOT:PSS-only electrode during the first and subsequent cycles (Figure S4). This suggests that the extent of lithium trapping due to the PEDOT:PSS in the first cycle is around $\sim 50 \mathrm{mAh} / \mathrm{g}$, somewhat less than the PEDOT:PSS described by Yao et al. $(\sim 100 \mathrm{mAh} / \mathrm{g})^{53}$ and the poly(phenanthrenequinone) based conductive binder/SiNP system described by Kim et al. $(\sim 87 \mathrm{mAh} / \mathrm{g}) \cdot{ }^{29}$ While we do not know the specific cause of this capacity loss associated with PEDOT:PSS, its contribution to the overall first cycle capacity loss is insignificant ( $1 \%$ for a $20 \mathrm{wt} \%$ composite).

Regarding the effect of the FA treatment, it is clear from Figure $2 b$ that the PEDOT:PSS/SiNP electrode with $10 \%$ FA treatment exhibits higher lithiation and delithiation capacities (3685 and $2858 \mathrm{~mA} \mathrm{~h} / \mathrm{g}$, respectively) compared to electrodes exposed to 0 or $5 \% \mathrm{FA}$; the first cycle delithiation capacity was $20 \%$ higher than the sample prepared without secondary doping treatment. In addition, we observe an increase in first cycle irreversible capacity (note: Table $\mathrm{S} 2$ in the Supporting Information provides the first cycle lithiation, delithiation and irreversible capacities when varying the amount of FA added). This confirms that electrodes prepared with optimum FA treatment results in a more efficient supporting matrix for the electrochemical utilization of the SiNPs. The enlarged lithiation profiles from inset in Figure $2 \mathrm{~b}$ also support this assertion; the long plateau for PEDOT:PSS/SiNP electrode with 10\% FA addition appeared 
at higher potential than other electrodes indicating that a relatively low cathodic over-potential was induced for PEDOT:PSS/SiNP with 10\% FA addition.

Figure 2c compares the cycling performance of these same electrodes. After 100 cycles at 1.0 $\mathrm{A} / \mathrm{g}$ it is found that conventional $\mathrm{CB} / \mathrm{Li}-\mathrm{PAA} / \mathrm{SiNP}$ electrode is inferior to all of the PEDOT:PSS-based electrodes; the silicon mass normalized capacity of CB/Li-PAA/SiNP electrode is markedly decreased from $2480 \mathrm{mAh} / \mathrm{g}$ ( $2^{\text {nd }}$ cycle) to $1510 \mathrm{mAh} / \mathrm{g}\left(100^{\text {th }}\right.$ cycle $)$. On the other hand, the PEDOT:PSS/SiNP electrode with 10\% FA treatment shows the highest specific capacity of $2681 \mathrm{mAh} / \mathrm{g}$ in the $2^{\text {nd }}$ cycle and $1950 \mathrm{mAh} / \mathrm{g}$ in the $100^{\text {th }}$ cycle.

Given the large difference in the electrical conductivity of these samples with differing FA treatments we also briefly examined their rate capabilities. Figure $2 d$ compares the $\mathrm{CB} / \mathrm{Li}$ PAA/SiNP electrode and PEDOT:PSS/SiNP electrodes with and without the optimized FA treatment when cycled at current densities from $0.5 \mathrm{~A} / \mathrm{g}$ to $5 \mathrm{~A} / \mathrm{g}$. Although all of the electrodes show similar specific capacities at a low current density of $0.5 \mathrm{~A} / \mathrm{g}$ (1-5 cycles), the specific capacity of both CB/Li-PAA/SiNP and the 5\% FA PEDOT:PSS/SiNP electrodes were significantly reduced for the higher current density $(1 \mathrm{~A} / \mathrm{g}$ to $5 \mathrm{~A} / \mathrm{g})$. In contrast, the $10 \% \mathrm{FA}-$ treated sample only suffered a $44 \%$ decrease in capacity when the current density was increase by a factor of $10(0.5$ to $5.0 \mathrm{~A} / \mathrm{g})$.

To support these measurements, EDX and Raman mapping was also carried out to investigate whether the presence of the FA alters the mixing of the PEDOT:PSS with the SiNPs. The EDX maps presented in the Supporting Information (Figure S5) indicate that the FA loading appears to have negligible effect on the mixing of the components, which is corroborated by the Raman maps for samples produced using a range of FA loadings (see Supporting Information, Figure S6-8).

Overall, these results demonstrate that the PEDOT:PSS-based bi-component electrode employing the optimized FA-secondary doping treatment provides more stable cycling retention and improved rate-capability compared with a conventional electrode with an equivalent SiNP loading. While this in situ FA treatment was employed with the sole intention of increasing the electrical conductivity of these composites, it is possible that there are secondary benefits associated with its inclusion. In particular, there is growing evidence that hydrogen bonding interactions between silanol functional group (present on the SiNP surface) and carboxylic acid moieties present on certain binders imparts an advantageous self-healing 
character to the overall electrode structure. ${ }^{9,54-56}$ This quality has been used to justify the promise of many polysaccharides as binders for silicon as well as synthetic polymers with high densities of carboxylic acid groups, such as PAA. ${ }^{8,21,57}$ A detailed investigation into this aspect of our FA-treated PEDOT:PSS/SiNP electrodes is an interesting subject for the further study of this system.

Effect of PEDOT:PSS loading on composite electrical properties: Using the optimized insitu FA loading of $10 \%$ we then prepared composite films on glass substrates with various PEDOT:PSS loadings (from 5 to $30 \mathrm{wt} \%$ ). These limits were determined by our ability to prepare slurry-cast films that remained mechanically robust at the lower range \& adherent to the substrate at the upper range (see Supporting Information, Figure S9). Regardless, these span active material loadings of general interest for Li-ion battery negative electrodes. Table S3 in the Supporing Information provides information on mass loading, thickness and density for the films described above.

Four-wire current-voltage and film profilometry measurements were performed on the resulting composite films and the electrical conductivity was found to vary from $\sigma_{D C}=0.7 \mathrm{~S} / \mathrm{cm}$ for the $5 \mathrm{wt} \%$ PEDOT:PSS sample up to $\sigma_{D C}=19 \mathrm{~S} / \mathrm{cm}$ for the $30 \mathrm{wt} \%$ sample (Figure 3a). It is known that the increased conductivity of an insulator upon the addition of a conducting filler can often be described by a power law arising from percolation theory; ${ }^{58} \sigma_{D C} \propto\left(M_{f}-M_{f, c}\right)^{n}$ . Although this expression should, strictly speaking, use volume fraction when describing the conductive filler content, here we have used the composite mass fraction $\left(M_{f}\right)$ for simplicity. In this expression, $M_{f, c}$ is the critical mass fraction where the first conductive pathway forms across the sample, beyond which, the further addition of conducting filler leads to a dramatic increase in conductivity according to the percolative exponent $n$. While the percolation threshold is certainly less than $5 \mathrm{wt} \%$ for this system, a more precise value cannot be determined due to the inability to produce samples with lower polymer loadings. However, when the data is approximately fitted using the universal value for the percolation exponent of $n=2.0$, the fit suggests that the percolation threshold is quite low $(\sim 1 \mathrm{wt} \%)$. In comparison, the electrical conductivity of the conventional electrode system had a conductivity of only 1.1 $\mathrm{mS} / \mathrm{cm}$, which is $2000 \times$ lower than the FA-treated PEDOT:PSS containing system at an equivalent SiNP loading ( $80 \mathrm{wt} \%$ ). However, we must note that the actually conductivity of 
the electrode may be somewhat less than these values during their operation at negative potential. $^{59}$

Effect of PEDOT:PSS loading on composite density and porosity: Following this, the PEDOT:PSS/SiNP samples with various conductive binder loadings were prepared identically on the $\mathrm{Cu}$ foil current collectors and the film density $(\rho)$ and porosity $(\varphi)$ were determined. Discs were cut from the main films and the composite mass and volume were measured. As shown in Figure $3 \mathrm{~b}$, the film density varied from $0.45 \mathrm{~g} / \mathrm{cm}^{3}$ for the $5 \mathrm{wt} \%$ sample up to 0.61 $\mathrm{g} / \mathrm{cm}^{3}$ for the $30 \mathrm{wt} \%$ sample. For comparison, a PEDOT:PSS-only film was produced separately by evaporative casting which was found to have a density of $1.2 \mathrm{~g} / \mathrm{cm}^{3}$. This value along with the density of bulk silicon $\left(\rho_{S i, b u l k}=2.3 \mathrm{~g} / \mathrm{cm}^{3}\right)$ was used to calculate the composite porosity as a function of PEDOT:PSS loading, shown in Figure 3c (see the Supporting Information for the expression for $\varphi$ ). Hence, there is a noticeable reduction on the overall film porosity from $\varphi=0.8$ to 0.66 over the composition range. Similar changes in film porosity were measured by Kim et al. when varying the composition of their poly(phenanthrenequinone)/SiNP composites over the same composition range. ${ }^{29}$

The presence of substantial free volume is made apparent by the SEM images for 10 and 30 wt\% samples shown in Figure 3d-g. While the appearance of PEDOT:PSS is difficult to discern for the lower PEDOT:PSS loading, for the $30 \mathrm{wt} \%$ sample a polymeric coating on the SiNPs is apparent. The presence of free volume within the film is important not only to allow electrolyte penetration within the electrode internal surfaces, but also to facilitate the expansion of silicon during lithiation. ${ }^{56,60}$ We note that because a volume increase of up to $~ 270 \%$ is expected for the SiNPs, the measured free volume is not sufficient to accommodate all of the swelling. This means the PEDOT:PSS network must withstand some mechanical extension during cycling. Future work will study the mechanical properties of the polymer in the presence of the electrolyte. In addition, while there is certainly scope to increase the film density (and hence the volumetric capacity) through calendaring, in this study, we have limited characterisation of the charge storage properties to the as-produced films. We note that Zhao et al. obtained optimal cycling performance for their polyfluorene-based conductive polymer/SiO electrodes when the porosity was reduced of 0.43 using calendering. ${ }^{36}$ This suggesting that improvements to our PEDOT:PSS/SiNP system could also be obtained through similar post-processing. 
Effect of PEDOT:PSS loading on composite electrochemical properties: Next we characterized the electrochemical performance of the PEDOT:PSS/SiNP electrodes when the PEDOT:PSS loading is varied from 5 to $30 \mathrm{wt} \%$. Here a combination of constant current and constant voltage $(\mathrm{CC}-\mathrm{CV})$ lithiation was used instead of the typical constant current (CC) mode, enabling investigation of the actual performances of the electrodes in the highly lithiated state. The resulting capacities as a function of cycle number under these conditions are shown in Figure 4 and the lithiation/delithiation profiles for all of the electrodes at cycles 1, 50 and 100 appear in the Supporting Information (Figure S10). By normalising the resulting lithiation capacities by the mass of the SiNPs only, it is clear that the addition of more conductive binder improves the performance of the available silicon (Figure $4 \mathrm{a}$ and $4 \mathrm{~b}$ ). Despite being unable to prepare samples with PEDOT:PSS loadings greater than $30 \mathrm{wt} \%$, the electrochemical utilization of the available silicon appears to be reaching a plateau at the higher loadings.

With the exception of the $5 \mathrm{wt} \%$ sample, all of the PEDOT:PSS containing samples provide greater silicon mass-normalized capacities than the CB/LiPAA-based control. The specific capacity of the electrodes were remarkably increased from 2071 to $2803 \mathrm{mAh} / \mathrm{g}$ at the $2^{\text {nd }}$ cycle by increasing the PEDOT:PSS content from 5 to $30 \mathrm{wt} \%$. For PEDOT:PSS contents $\geq 20 \mathrm{wt} \%$ the resulting specific capacity values approach $2000 \mathrm{mAh} / \mathrm{g}$ even following 100 charge/discharge cycles; 1927, 1985, and $2052 \mathrm{mAh} / \mathrm{g}$ for 20, 25, and $30 \mathrm{wt} \%$ PEDOT:PSS containing electrodes, respectively. The Figure $4 \mathrm{c}$ inset provides the capacity retention at the $100^{\text {th }}$ cycle relative to the capacity at the $2^{\text {nd }}$ cycle, which indicates that for all electrodes with PEDOT:PSS contents $\geq 15 \mathrm{wt} \%$ the retention is $\sim 75 \%$. These are extremely promising results among the Si-based electrodes and are an exciting demonstration of the excellent performance that can be achieved using electrodes that consist of commercially available 'off-the-shelf' components and industrially scalable fabrication processes; this is a stark contrast with other high performance though synthetically complex conducting polymer binders that emphasize the need for specifically tailored functionality. ${ }^{30,34-37}$

While it is important to know the specific capacity that is solely associated with the silicon, the capacity normalized to the total electrode mass is of far greater practical importance. This data is shown Figure $4 \mathrm{c}$ as a function of cycle number and as a function of PEDOT:PSS loading for different cycle numbers in Figure $4 \mathrm{~d}$ (note that $M_{\text {Total }}=M_{S i}+M_{\text {PEDOT:PSS). Given the very low }}$ specific capacity of PEDOT:PSS alone (<20 mAh/g, see Supporting Information, Figure S3), it is apparent that $C / M_{T}$ does not vary with PEDOT:PSS loading as per the rule of mixtures. 
Rather, a capacity maximum is observed for the $20 \mathrm{wt} \%$ PEDOT:PSS composite, which provides a total mass normalized capacity of $2167 \mathrm{mAh} / \mathrm{g}$ at the $2^{\text {nd }}$ cycle and $1542 \mathrm{mAh} / \mathrm{g}$ at the $100^{\text {th }}$ cycle. In contrast, Liu et al. found that the performance of their conductive binder/SiNP system was optimal when using $30 \mathrm{wt} \%$ non-active components, ${ }^{35}$ and for conventional (3-component) Si-based systems it is common for over $30 \mathrm{wt} \%$ of inactive components to be used. ${ }^{61,62}$ Where possible, it is desirable to minimize the content of nonactive constituents within the electrode in order to maximize overall capacity. In this case, when using just $10 \mathrm{wt} \%$ non-active components, this system was able to provide $2131 \mathrm{mAh} / \mathrm{g}$ at the $2^{\text {nd }}$ cycle and 1332 at the $100^{\text {th }}$ cycle. These results highlight the great utility of partnering silicon with a single conductive binder in order to reduce the presence of non-active components within the electrode structure.

Further characterisation of the $20 \mathrm{wt} \%$ PEDOT:PSS/SiNP sample was carried out to investigate the effect of the areal loading $(M / A)$ and the applied current density $(I / M)$ on performance. Figure $5 \mathrm{a}$ and $5 \mathrm{~b}$ presents the areal capacity and silicon mass normalized capacity for areal loadings ranging from $M / A=0.4 \mathrm{mg} / \mathrm{cm}^{2}$ to $1.5 \mathrm{mg} / \mathrm{cm}^{2}$. Table S4 in the Supporting Information details the mass loading, thickness and density of these samples. As indicated by the Figure 5a inset, $C / A$ scales linearly with mass loading, indicating the entire electrode is being utilized within this thickness range. This is likely a consequence of the excellent electrical properties of these electrodes, enabling the utilisation of silicon far from the current collector as well as of the electrode porosity which facilitates deep electrolyte penetration. This is also made apparent by the data shown in Figure $5 \mathrm{~b}$ whereby the $C / M_{S i}$ values essentially overlap regardless of their differing areal loadings. The thickest sample that was tested $(M / A \sim 1.5$ $\mathrm{mg} / \mathrm{cm}^{2}$ ) provided an areal capacity of $\sim 3 \mathrm{mAh} / \mathrm{cm}^{2}$, which meets the capacity requirement for the negative electrode in the commercialized LiB cell configuration. The preparation of films with areal loadings greater than $M / A=1.5 \mathrm{mg} / \mathrm{cm}^{2}$ was problematic due to a loss of adherence between the composite and the $\mathrm{Cu}$ foil substrate during film drying.

Although areal capacities for experimental Si-based systems are typically less than $\sim 1$ $\mathrm{mAh} / \mathrm{cm}^{2}$, some other exceptions exist; Zhao et al. demonstrated $C / A=3.5 \mathrm{mAh} / \mathrm{cm}^{2}$ for their calendered polyfluorene-based conductive polymer/SiO electrodes,${ }^{36}$ Liu et al. prepared a Sibased system capable of storing $3.7 \mathrm{mAh} / \mathrm{cm}^{2}$ (albeit with a fairly low electrode density; $\rho \sim 0.4$ $\mathrm{mg} / \mathrm{cm}^{3}$ and $\left.\mathrm{t} \sim 120 \mu \mathrm{m}\right) .{ }^{63}$ Probably the highest areal capacity to date was achieved by Song $e t$ al. with their PAA/PVA mixed binder system, measuring $\mathrm{C} / \mathrm{A}=4.3 \mathrm{mAh} / \mathrm{cm}^{2}{ }^{64}$ However, this 
composite employed $40 \mathrm{wt} \%$ non-active components with the SiNP active material, including $10 \mathrm{wt} \%$ carbon fibres to enable the preparation of these high mass loading electrodes. Based on previous studies on supercapacitors ${ }^{65}$ and hydrogen evolution electrocatalysts, ${ }^{66}$ we believe that the capacity of our PEDOT:PSS/SiNP could be increased significantly through the inclusion of small loadings of SWNTs $(\sim 5 \mathrm{wt} \%)$. This would enable the preparation of far thicker films that remain both robust and adherent to the substrate, giving significant enhancements in $\mathrm{C} / \mathrm{A}$.

Figure $5 \mathrm{c}$ and $5 \mathrm{~d}$ presents the silicon normalized specific capacity and the corresponding capacity retention for the $20 \mathrm{wt} \%$ PEDOT:PSS/SiNP electrodes under different applied current densities. For the lowest current $(I / M=0.5 \mathrm{~A} / \mathrm{g})$ a very high specific capacity over $3000 \mathrm{mAh} / \mathrm{g}$ is found during the initial cycles and is $2186 \mathrm{mAh} / \mathrm{g}$ is retained after 100 cycles. When increasing the current density, the electrodes exhibit lower specific capacity but higher capacity retention, which is a common tendency associated with the depth of lithiation and the related structural transition of the silicon. At the highest current density $(I / M=10 \mathrm{~A} / \mathrm{g})$, very high capacity retention is observed, up to $95 \%$ after 100 cycles. The observed increase in capacity with cycle number is frequently observed in the literature when applying very fast charging/discharging condition, which could be due to delayed wetting of electrolyte into the electrode. $^{32}$

To further investigate the long-term cycling stability of these PEDOT:PSS/SiNP electrodes rapid charge and discharge measurements were carried out over 500 cycles. As shown in the Supporting Information (Figure S11), two separate samples were cycled under different conditions; one at various current densities from 3 to $10 \mathrm{~A} / \mathrm{g}$ and another at a fixed current density of $10 \mathrm{~A} / \mathrm{g}$. For the latter sample, a specific capacity of $436 \mathrm{mAh} / \mathrm{g}$ was retained after 500 cycles. In Figure 5e we have plotted the total electrode capacity of these samples as a function of the applied current density (at cycle 100), as well as a range of data from the literature (note: Figure S12 in the Supporting Information reproduces this figure along with a detailed legend that refers to Table S5). While our electrodes are very competitive at lower current densities, unfortunately, it was difficult to find other systems with which to compare at higher current densities $(I / M>1 \mathrm{~A} / \mathrm{g})$.

To put the excellent performance of this work into a broad perspective, Figure $5 \mathrm{f}$ presents a detailed literature comparison of competitive silicon-containing electrode systems when 
capacity is normalized to the mass of active materials as well as the total electrode mass. We emphasize that it is difficult to make a direct comparison between different systems due to the large number of variables present (regarding both compositional and testing conditions). As such, we have classified the literature values into two categories based on the total electrode areal loading $(M / A)$ as well as the wt $\%$ of silicon and the relevant cycle number (note: this data is reproduced in the Supporting Information, Table S5). Our PEDOT:PSS/SiNP system performs better than all other samples in the high areal loading category $\left(M / A>1.0 \mathrm{mg} / \mathrm{cm}^{2}\right)$ other than the work of Zhang et al. whom used ionically crosslinked alginate as a binder. However, we note that these electrodes were tested at a lower current density than our system $(I / M=0.42 \mathrm{~A} / \mathrm{g}$ compared with $0.5 \mathrm{~A} / \mathrm{g})$ and as such these two systems are, in fact, very similar. This clearly shows that using PEDOT:PSS as both mechanical binder and electrical additive results in Si-based negative electrodes with state of the art performance.

\section{CONCLUSIONS}

In conclusion, we have investigated the properties $\mathrm{LiB}$ negative electrodes consisting of silicon nanoparticles partnered with PEDOT:PSS, which here acts as a conductive binder. Not only does this dual combination provide excellent performance but also presents a reduced parameter space by eliminating the need to tailor the interaction between the active material and an additional conductive additive via a polymeric binder. In fact, we feel this is the most important advantage of this system in light of the particularly demanding set of requirements for the binder component of large volume change electrodes. That these two components are commercially available in large quantities and processable very easily by conventional means (i.e. slurry casting using water) is also a very attractive feature of this work.

This system presents many opportunities for further studies, which will almost certainly lead to improvements in performance, as well as a deeper understand of the factors responsible for the success of this particular combination. PEDOT:PSS is not elastomeric and also does not possess the carboxylic acid functional groups required for the so-called 'self-healing' character of other successful binder systems. As such, it will be interesting to investigate whether the formic acid that was added during electrode fabrication has benefits in addition to just improving the electrical conductivity of the PEDOT:PSS. In addition, as these electrodes were characterized as prepared, future work will needed to study the effect of calendering (and hence reduced electrode porosity) on both cyclability and volumetric capacity. 
In this study we have made no attempt to investigate the nature of the SEI layer that forms on PEDOT:PSS coated SiNPs. In fact, we feel that optimisation of this SEI layer is the most promising avenue for achieving further improvements to this system. While electrodes with relatively high loadings of active material $(80 \mathrm{wt} \%)$ was optimal, those prepared with $90 \mathrm{wt} \%$ silicon also performed quite well. As such, the addition of small quantities of other polymers could be used to modify the interfacial properties of the silicon with the binder matrix or the mechanical properties of the PEDOT:PSS.

Overall, this system very effectively highlights the merits of combining SiNP with PEDOT:PSS as a conductive binder and provides a valuable new baseline for further studies involving the use of this commercially available conductive polymer to alleviate the problems associated with large volume change LiB negative electrodes.

\section{METHODS}

Silicon Nanoparticle Characterisation: The shape and crystallinity of the SiNPs were examined with a transmission electron microscope (FEI Titan) operated at $300 \mathrm{keV}$. To prepare the sample for imaging a dispersion of SiNPs in water was drop-cast onto a Lacey Carbon grid and baked overnight at $100^{\circ} \mathrm{C}$ under vacuum. X-ray photoelectron spectroscopy was measured for powder samples using monochromated Al Ka X-rays from an Omicron XM1000 MkII Xray source and an Omicron EA125 energy analyzer. The analyzer pass energy was set to 15 $\mathrm{eV}$. After subtraction of a Shirley background the Si $2 \mathrm{p}$ core-level spectra were fitted with Gaussian-Lorentzian line shapes using the software CasaXPS.

Electrode Preparation: PEDOT:PSS (Clevios PH 1000) was received from Heraeus as an aqueous solution (concentration $=11 \mathrm{mg} / \mathrm{mL}$ ). SiNPs were obtained as a powder from US Research Nanomaterials (mean diameter: $50-70 \mathrm{~nm}$, laser synthesized) while formic acid (FA) was purchased from Sigma Aldrich. These were combined in a mortar and pestle and stirred for 30 minutes to form a slurry. The PEDOT:PSS loading was varied between 5 and $30 \mathrm{wt} \%$ (note: the sample compositions are provided in the Supporting Information, Tables S1, S3, and S4). We note that FA was directly added into the Si/PEDOT:PSS slurry as a secondary dopant to modify the PEDOT:PSS conductivity. The optimum loading of FA (10\% relative to the volume of PEDOT:PSS solution) was determined in a separate set of experiments. Isopropyl alcohol (IPA, Aldrich) was also added dropwise to the mortar during the mixing process to modify the viscosity as required. The resulting slurries were cast into films on $\mathrm{Cu}$ foil substrates 
(MTI Corp., thickness $=12 \mu \mathrm{m}$ ) using a doctor blade apparatus. The doctor blade clearance was varied from 20 and 100 microns to vary the thickness of the produced films. The films were then heated at $T=110^{\circ} \mathrm{C}$ under vacuum for 1 hour to remove residual water and FA (note: the boiling point of FA at standard pressure is $100.8^{\circ} \mathrm{C}$ ).

For comparison, a conventional composite electrode system was prepared in the same manner. This consisted of carbon black powder (Timical Super C65, MTI Corp.) and Li-PAA binder prepared via the method described by reported by Eberma et al., ${ }^{67}$ which were mixed with the same SiNPs as for the experimental system in a fixed ratio of $80 \mathrm{wt} \% \mathrm{Si}, 10 \mathrm{wt} \% \mathrm{Li}-\mathrm{PAA}$ and $10 \mathrm{wt} \% \mathrm{CB}$.

Electrode Characterisation: The morphologies and microstructure of the samples were examined using FE-SEM (Supra 40, Carl Zeiss) with an attached energy dispersive X-ray spectrometry system (INCA EDS, Oxford Instruments). The film density was calculated by first punching a number of circular discs with diameter $=12 \mathrm{~mm}$ from the main film with the area over $100 \mathrm{~cm}^{2}$. The mass $(M)$ and thickness $(t)$ of each disc was determined using a microbalance and micrometre and these were used to calculate the film density $\left(\rho=M(A t)^{-1}\right.$ ). The thickness and mass of $\mathrm{Cu}$ foil substrate was determined in the same manner and subtracted in each case. The areal loading of coated material was approximately $1 \mathrm{mg} / \mathrm{cm}^{2}$ prior to thickness dependence studies following.

The four-point probe technique was used to investigate the DC electrical conductivity of the samples with different conditions and compositions. Here samples were prepared identically as described above though with non-conductive glass plate used in place of the $\mathrm{Cu}$ foil. Four parallel electrode contacts were created on the film surface using conductive silver paint (Agar Scientific). The electrode separation and width were $\sim 1 \mathrm{~cm}$ and $\sim 0.5 \mathrm{~cm}$, respectively. The resistance of each sample was calculated from current-voltage curves measured using a Keithley 2400 source meter. The electrode thickness was determined by contact profilometry (Dekat 6M, Veeco Instruments). Here scratches were created using a scribe and the mean film thickness was calculated from nine separated lateral profiles obtained for different regions on the film surface.

Raman spectra were acquired using a Witec Alpha $300 \mathrm{R}$ with a $532 \mathrm{~nm}$ excitation laser and a spectral grating with 600 lines/mm. Raman maps were generated for the PEDOT:PSS/SiNP samples by taking $80 \times 80$ discrete point spectra, with an integration time of $0.2 \mathrm{~s}$ per spectrum, 
over a $20 \times 20 \mu \mathrm{m}$ area. This was done using a $100 \times$ objective lens and a laser power of $\sim 30$ $\mu \mathrm{W}$. This low power was necessary for these composite samples as when powers $>100 \mu \mathrm{W}$ were used a disordered carbon signal was observed rather that the well-known PEDOT signal, suggesting that the laser was damaging the PEDOT:PSS. Average spectra for PEDOT only films were calculated from line scans consisting of 10 discrete point spectra. These were acquired using a $20 \times$ objective with a laser power of $\sim 600 \mu \mathrm{W}$.

The electrochemical properties of PEDOT:PSS/SiNP electrodes were investigated using 2032type coin cells (MTI Corp.). A lithium metal disc with diameter $=16 \mathrm{~mm}$ was used as the counter electrode, and $1 \mathrm{M} \mathrm{LiPF}_{6}$ in ethyl carbonate (EC)/dimethyl carbonate (DEC)/fluoroethylene carbonate (FEC) $(3: 6: 1 \mathrm{v} / \mathrm{v})$ was used as the electrolyte. Each working electrode was punched from the main film into discs with diameter $=12 \mathrm{~mm}$. A Celgard 2320 separator was used for all samples. The electrochemical properties of the electrodes were measured within a voltage range of 1.2-0.005 V using constant current (CC) mode or constant current-constant voltage (CC-CV) mode using a potentiostat/galvanostat (VMP3, Bio-logic). In the $\mathrm{CC}-\mathrm{CV}$ mode, the electrodes were first lithiated at $1 \mathrm{~A} / \mathrm{g}$ and held at $0.001 \mathrm{~V}$ until the current had decayed to $0.1 \mathrm{~A} / \mathrm{g}$. Then the electrodes were delithiated at a $1 \mathrm{~A} / \mathrm{g}$ until a $1.2 \mathrm{~V}$ cut-off was reached. The specific capacities of the electrodes were normalized to either the mass of active material (SiNP) or the total mass of electrode components (combined SiNP and PEDOT:PSS mass) to compare the performances. Electrochemical impedance spectroscopy was performed using an impedance analyser at a DC bias voltage of $0.2 \mathrm{~V}$ and AC frequencies ranging from $200 \mathrm{kHz}$ to $10 \mathrm{mHz}$. Immediately prior to acquiring the EIS spectra working electrodes were conditioned at the as-mentioned DC bias for 2 hours. 
Supporting Information Available: Detailed methods, materials characterisation and literature review. This material is available free of charge via the Internet at http://pubs.acs.org.

\section{Acknowledgements}

This publication has emanated from research conducted with the financial support of Science Foundation Ireland (SFI) under Grant Number SFI/12/RC/2278. JNC and VN are very grateful to Bell Labs Ireland for continuing support.

\section{REFERENCES}

1. $\quad$ Armand, M.; Tarascon, J. M., Building Better Batteries. Nature 2008, 451, 652-657.

2. Thackeray, M. M.; Wolverton, C.; Isaacs, E. D., Electrical Energy Storage for TransportationApproaching the Limits of, and Going Beyond, Lithium-Ion Batteries. Energy Environ. Sci. 2012, 5, 7854-7863.

3. $\quad$ Bonaccorso, F.; Colombo, L.; Yu, G.; Stoller, M.; Tozzini, V.; Ferrari, A. C.; Ruoff, R. S.; Pellegrini, V., Graphene, Related Two-Dimensional Crystals, and Hybrid Systems for Energy Conversion and Storage. Science 2015, 347.

4. Hassoun, J.; Bonaccorso, F.; Agostini, M.; Angelucci, M.; Betti, M. G.; Cingolani, R.; Gemmi, M.; Mariani, C.; Panero, S.; Pellegrini, V.; Scrosati, B., An Advanced Lithium-Ion Battery Based on a Graphene Anode and a Lithium Iron Phosphate Cathode. Nano Lett. 2014, 14, 4901-4906.

5. Wang, H.; Yang, Y.; Liang, Y.; Cui, L.-F.; Casalongue, H. S.; Li, Y.; Hong, G.; Cui, Y.; Dai, H., Limn1-Xfexpo4 Nanorods Grown on Graphene Sheets for Ultrahigh-Rate-Performance Lithium Ion Batteries. Angew. Chem.,Int. Ed. 2011, 50, 7364-7368.

6. Wang, H.; Yang, Y.; Liang, Y.; Robinson, J. T.; Li, Y.; Jackson, A.; Cui, Y.; Dai, H., GrapheneWrapped Sulfur Particles as a Rechargeable Lithium-Sulfur Battery Cathode Material with High Capacity and Cycling Stability. Nano Lett. 2011, 11, 2644-2647.

7. Beaulieu, L. Y.; Hatchard, T. D.; Bonakdarpour, A.; Fleischauer, M. D.; Dahn, J. R., Reaction of Li with Alloy Thin Films Studied by in Situ Afm. J. Electrochem. Soc. 2003, 150, A1457-A1464.

8. $\quad$ Erk, C.; Brezesinski, T.; Sommer, H.; Schneider, R.; Janek, J., Toward Silicon Anodes for Next-Generation Lithium Ion Batteries: A Comparative Performance Study of Various Polymer Binders and Silicon Nanopowders. ACS Appl. Mater. Interfaces 2013, 5, 7299-7307.

9. Jeong, Y. K.; Kwon, T.-w.; Lee, I.; Kim, T.-S.; Coskun, A.; Choi, J. W., Millipede-Inspired Structural Design Principle for High Performance Polysaccharide Binders in Silicon Anodes. Energy Environ. Sci. 2015, 8, 1224-1230.

10. Timmons, A.; Dahn, J. R., In Situ Optical Observations of Particle Motion in Alloy Negative Electrodes for Li-Ion Batteries. J. Electrochem. Soc. 2006, 153, A1206-A1210.

11. Nie, M.; Abraham, D. P.; Chen, Y.; Bose, A.; Lucht, B. L., Silicon Solid Electrolyte Interphase (Sei) of Lithium Ion Battery Characterized by Microscopy and Spectroscopy. The Journal of Physical Chemistry C 2013, 117, 13403-13412.

12. Pinson, M. B.; Bazant, M. Z., Theory of Sei Formation in Rechargeable Batteries: Capacity Fade, Accelerated Aging and Lifetime Prediction. J. Electrochem. Soc. 2013, 160, A243-A250.

13. Coleman, J. N.; Lotya, M.; O'Neill, A.; Bergin, S. D.; King, P. J.; Khan, U.; Young, K.; Gaucher, A.; De, S.; Smith, R. J.; Shvets, I. V.; Arora, S. K.; Stanton, G.; Kim, H.-Y.; Lee, K.; Kim, G. 
T.; Duesberg, G. S.; Hallam, T.; Boland, J. J.; Wang, J. J., et al., Two-Dimensional Nanosheets Produced by Liquid Exfoliation of Layered Materials. Science 2011, 331, 568-571.

14. Choi, N.-S.; Yew, K. H.; Choi, W.-U.; Kim, S.-S., Enhanced Electrochemical Properties of a Si-Based Anode Using an Electrochemically Active Polyamide Imide Binder. J. Power Sources 2008, 177, 590-594.

15. Chan, C. K.; Peng, H.; Liu, G.; Mcllwrath, K.; Zhang, X. F.; Huggins, R. A.; Cui, Y., HighPerformance Lithium Battery Anodes Using Silicon Nanowires. Nat. Nanotechnol. 2008, 3, 31-35.

16. Liang, B.; Liu, Y.; Xu, Y., Silicon-Based Materials as High Capacity Anodes for Next Generation Lithium Ion Batteries. J. Power Sources 2014, 267, 469-490.

17. Liu, X. H.; Zhong, L.; Huang, S.; Mao, S. X.; Zhu, T.; Huang, J. Y., Size-Dependent Fracture of Silicon Nanoparticles During Lithiation. ACS Nano 2012, 6, 1522-1531.

18. Chen, Z.; Christensen, L.; Dahn, J. R., Large-Volume-Change Electrodes for Li-Ion Batteries of Amorphous Alloy Particles Held by Elastomeric Tethers. Electrochem. Commun. 2003, 5, 919-923. 19. Chen, Z.; Christensen, L.; Dahn, J. R., Comparison of Pvdf and Pvdf-Tfe-P as Binders for Electrode Materials Showing Large Volume Changes in Lithium-Ion Batteries. J. Electrochem. Soc. 2003, 150, A1073-A1078.

20. Garsuch, R. R.; Le, D.-B.; Garsuch, A.; Li, J.; Wang, S.; Farooq, A.; Dahn, J. R., Studies of Lithium-Exchanged Nafion as an Electrode Binder for Alloy Negatives in Lithium-Ion Batteries. $J$. Electrochem. Soc. 2008, 155, A721-A724.

21. Magasinski, A.; Zdyrko, B.; Kovalenko, I.; Hertzberg, B.; Burtovyy, R.; Huebner, C. F.; Fuller, T. F.; Luzinov, I.; Yushin, G., Toward Efficient Binders for Li-Ion Battery Si-Based Anodes: Polyacrylic Acid. ACS Appl. Mater. Interfaces 2010, 2, 3004-3010.

22. Kim, J. S.; Choi, W.; Cho, K. Y.; Byun, D.; Lim, J.; Lee, J. K., Effect of Polyimide Binder on Electrochemical Characteristics of Surface-Modified Silicon Anode for Lithium Ion Batteries. J. Power Sources 2013, 244, 521-526.

23. Buqa, H.; Holzapfel, M.; Krumeich, F.; Veit, C.; Novák, P., Study of Styrene Butadiene Rubber and Sodium Methyl Cellulose as Binder for Negative Electrodes in Lithium-Ion Batteries. J. Power Sources 2006, 161, 617-622.

24. Kovalenko, I.; Zdyrko, B.; Magasinski, A.; Hertzberg, B.; Milicev, Z.; Burtovyy, R.; Luzinov, I.; Yushin, G., A Major Constituent of Brown Algae for Use in High-Capacity Li-Ion Batteries. Science 2011, 334, 75-79.

25. Jeong, Y. K.; Kwon, T.-w.; Lee, I.; Kim, T.-S.; Coskun, A.; Choi, J. W., Hyperbranched BCyclodextrin Polymer as an Effective Multidimensional Binder for Silicon Anodes in Lithium Rechargeable Batteries. Nano Lett. 2014, 14, 864-870.

26. Liu, W.-R.; Yang, M.-H.; Wu, H.-C.; Chiao, S. M.; Wu, N.-L., Enhanced Cycle Life of Si Anode for Li-Ion Batteries by Using Modified Elastomeric Binder. Electrochem. Solid-State Lett. 2005, 8, A100-A103.

27. Jeena, M. T.; Lee, J.-I.; Kim, S. H.; Kim, C.; Kim, J.-Y.; Park, S.; Ryu, J.-H., Multifunctional Molecular Design as an Efficient Polymeric Binder for Silicon Anodes in Lithium-Ion Batteries. ACS Appl. Mater. Interfaces 2014, 6, 18001-18007.

28. Hochgatterer, N. S.; Schweiger, M. R.; Koller, S.; Raimann, P. R.; Wöhrle, T.; Wurm, C.; Winter, M., Silicon/Graphite Composite Electrodes for High-Capacity Anodes: Influence of Binder Chemistry on Cycling Stability. Electrochem. Solid-State Lett. 2008, 11, A76-A80.

29. Kim, S.-M.; Kim, M. H.; Choi, S. Y.; Lee, J. G.; Jang, J.; Lee, J. B.; Ryu, J. H.; Hwang, S. S.; Park, J.-H.; Shin, K.; Kim, Y. G.; Oh, S. M., Poly(Phenanthrenequinone) as a Conductive Binder for Nano-Sized Silicon Negative Electrodes. Energy Environ. Sci. 2015, 8, 1538-1543.

30. Zhao, H.; Wang, Z.; Lu, P.; Jiang, M.; Shi, F.; Song, X.; Zheng, Z.; Zhou, X.; Fu, Y.; Abdelbast, G.; Xiao, X.; Liu, Z.; Battaglia, V. S.; Zaghib, K.; Liu, G., Toward Practical Application of Functional Conductive Polymer Binder for a High-Energy Lithium-Ion Battery Design. Nano Lett. 2014, 14, 67046710 .

31. Sengodu, P.; Deshmukh, A. D., Conducting Polymers and Their Inorganic Composites for Advanced Li-Ion Batteries: A Review. RSC Adv. 2015, 5, 42109-42130.

32. Wu, H.; Yu, G.; Pan, L.; Liu, N.; McDowell, M. T.; Bao, Z.; Cui, Y., Stable Li-Ion Battery Anodes by in-Situ Polymerization of Conducting Hydrogel to Conformally Coat Silicon Nanoparticles. Nat Commun 2013, 4. 
33. Park, S.-J.; Zhao, H.; Ai, G.; Wang, C.; Song, X.; Yuca, N.; Battaglia, V. S.; Yang, W.; Liu, G., Side-Chain Conducting and Phase-Separated Polymeric Binders for High-Performance Silicon Anodes in Lithium-Ion Batteries. J. Am. Chem. Soc. 2015, 137, 2565-2571.

34. Wu, M.; Xiao, X.; Vukmirovic, N.; Xun, S.; Das, P. K.; Song, X.; Olalde-Velasco, P.; Wang, D.; Weber, A. Z.; Wang, L.-W.; Battaglia, V. S.; Yang, W.; Liu, G., Toward an Ideal Polymer Binder Design for High-Capacity Battery Anodes. J. Am. Chem. Soc. 2013, 135, 12048-12056.

35. Liu, G.; Xun, S.; Vukmirovic, N.; Song, X.; Olalde-Velasco, P.; Zheng, H.; Battaglia, V. S.; Wang, L.; Yang, W., Polymers with Tailored Electronic Structure for High Capacity Lithium Battery Electrodes. Adv. Mater. 2011, 23, 4679-4683.

36. Zhao, H.; Yuca, N.; Zheng, Z.; Fu, Y.; Battaglia, V. S.; Abdelbast, G.; Zaghib, K.; Liu, G., High Capacity and High Density Functional Conductive Polymer and Sio Anode for High-Energy Lithium-Ion Batteries. ACS Appl. Mater. Interfaces 2015, 7, 862-866.

37. Xun, S.; Xiang, B.; Minor, A.; Battaglia, V.; Liu, G., Conductive Polymer and Silicon Composite Secondary Particles for a High Area-Loading Negative Electrode. J. Electrochem. Soc. 2013, 160, A1380-A1383.

38. Yao, Y.; Liu, N.; McDowell, M. T.; Pasta, M.; Cui, Y., Improving the Cycling Stability of Silicon Nanowire Anodes with Conducting Polymer Coatings. Energy Environ. Sci. 2012, 5, 79277930.

39. Chen, Z.; To, J. W. F.; Wang, C.; Lu, Z.; Liu, N.; Chortos, A.; Pan, L.; Wei, F.; Cui, Y.; Bao, Z., A Three-Dimensionally Interconnected Carbon Nanotube-Conducting Polymer Hydrogel Network for High-Performance Flexible Battery Electrodes. Advanced Energy Materials 2014, 4, n/a-n/a.

40. Yue, L.; Wang, S.; Zhao, X.; Zhang, L., Nano-Silicon Composites Using Poly(3,4Ethylenedioxythiophene): Poly(Styrenesulfonate) as Elastic Polymer Matrix and Carbon Source for Lithium-Ion Battery Anode. J. Mater. Chem. 2012, 22, 1094-1099.

41. Sun, K.; Zhang, S.; Li, P.; Xia, Y.; Zhang, X.; Du, D.; Isikgor, F. H.; Ouyang, J., Review on Application of Pedots and Pedot:Pss in Energy Conversion and Storage Devices. J. Mater. Sci.: Mater. Electron. 2015, 26, 4438-4462.

42. $\quad$ Courtel, F. M.; Niketic, S.; Duguay, D.; Abu-Lebdeh, Y.; Davidson, I. J., Water-Soluble Binders for Mcmb Carbon Anodes for Lithium-Ion Batteries. J. Power Sources 2011, 196, 2128-2134. 43. Roncali, J.; Blanchard, P.; Frère, P., 3,4-Ethylenedioxythiophene (Edot) as a Versatile Building Block for Advanced Functional П-Conjugated Systems. J. Mater. Chem. 2005, 15, 1589-1610.

44. Lipomi, D. J.; Lee, J. A.; Vosgueritchian, M.; Tee, B. C. K.; Bolander, J. A.; Bao, Z., Electronic Properties of Transparent Conductive Films of Pedot:Pss on Stretchable Substrates. Chem. Mater. 2012, 24, 373-382.

45. Higgins, T. M.; Coleman, J. N., Avoiding Resistance Limitations in High-Performance Transparent Supercapacitor Electrodes Based on Large-Area, High-Conductivity Pedot:Pss Films. ACS Appl. Mater. Interfaces 2015, 7, 16495-16506.

46. McCarthy, J. E.; Hanley, C. A.; Brennan, L. J.; Lambertini, V. G.; Gun'ko, Y. K., Fabrication of Highly Transparent and Conducting Pedot:Pss Films Using a Formic Acid Treatment. J. Mater. Chem. C 2013, 2, 764-770.

47. Wu, H.; Chan, G.; Choi, J. W.; Ryu, I.; Yao, Y.; McDowell, M. T.; Lee, S. W.; Jackson, A.; Yang, Y.; Hu, L.; Cui, Y., Stable Cycling of Double-Walled Silicon Nanotube Battery Anodes through Solid-Electrolyte Interphase Control. Nat. Nanotechnol. 2012, 7, 309-314.

48. Kim, Y. H.; Sachse, C.; Machala, M. L.; May, C.; Müller-Meskamp, L.; Leo, K., Highly Conductive Pedot:Pss Electrode with Optimized Solvent and Thermal Post-Treatment for Ito-Free Organic Solar Cells. Adv. Funct. Mater. 2011, 21, 1076-1081.

49. Nardes, A. M.; Kemerink, M.; de Kok, M. M.; Vinken, E.; Maturova, K.; Janssen, R. A. J., Conductivity, Work Function, and Environmental Stability of Pedot:Pss Thin Films Treated with Sorbitol. Organic Electronics 2008, 9, 727-734.

50. Xia, Y.; Sun, K.; Ouyang, J., Solution-Processed Metallic Conducting Polymer Films as Transparent Electrode of Optoelectronic Devices. Adv. Mater. 2012, 24, 2436-2440.

51. Nardes, A. M.; Janssen, R. A. J.; Kemerink, M., A Morphological Model for the SolventEnhanced Conductivity of Pedot:Pss Thin Films. Adv. Funct. Mater. 2008, 18, 865-871.

52. Groenendaal, B. L.; Jonas, F.; Freitag, D.; Pielartzik, H.; Reynolds, J. R., Poly(3,4Ethylenedioxythiophene) and Its Derivatives: Past, Present, and Future. Adv. Mater. 2000, 12, 481-494. 
53. Yao, Y.; Liu, N.; McDowell, M. T.; Pasta, M.; Cui, Y., Improving the Cycling Stability of Silicon Nanowire Anodes with Conducting Polymer Coatings. Energy and Environmental Science 2012, 5, 7927-7930.

54. Wang, C.; Wu, H.; Chen, Z.; McDowell, M. T.; Cui, Y.; Bao, Z., Self-Healing Chemistry Enables the Stable Operation of Silicon Microparticle Anodes for High-Energy Lithium-Ion Batteries. Nature Chem. 2013, 5, 1042-1048.

55. Kwon, T.-w.; Jeong, Y. K.; Lee, I.; Kim, T.-S.; Choi, J. W.; Coskun, A., Systematic MolecularLevel Design of Binders Incorporating Meldrum's Acid for Silicon Anodes in Lithium Rechargeable Batteries. Adv. Mater. 2014, 26, 7979-7985.

56. Guo, J.; Wang, C., A Polymer Scaffold Binder Structure for High Capacity Silicon Anode of Lithium-Ion Battery. Chem. Commun. 2010, 46, 1428-1430.

57. Komaba, S.; Shimomura, K.; Yabuuchi, N.; Ozeki, T.; Yui, H.; Konno, K., Study on Polymer Binders for High-Capacity Sio Negative Electrode of Li-Ion Batteries. J. Phys. Chem. C 2011, 115, 13487-13495.

58. Stauffer, D.; Aharony, A., Introduction to Percolation Theory. Taylor \& Francis: 1994; p 205.

59. Ahonen, H. J.; Lukkari, J.; Kankare, J., N- and P-Doped Poly(3,4-Ethylenedioxythiophene): Two Electronically Conducting States of the Polymer. Macromolecules 2000, 33, 6787-6793.

60. Han, Z.-J.; Yabuuchi, N.; Shimomura, K.; Murase, M.; Yui, H.; Komaba, S., High-Capacity Si-Graphite Composite Electrodes with a Self-Formed Porous Structure by a Partially Neutralized Polyacrylate for Li-Ion Batteries. Energy Environ. Sci. 2012, 5, 9014.

61. Bridel, J. S.; Azaïs, T.; Morcrette, M.; Tarascon, J. M.; Larcher, D., Key Parameters Governing the Reversibility of Si/Carbon/Cmc Electrodes for Li-Ion Batteries. Chem. Mater. 2010, 22, 1229-1241. 62. Beattie, S. D.; Larcher, D.; Morcrette, M.; Simon, B.; Tarascon, J. M., Si Electrodes for Li-Ion Batteries-a New Way to Look at an Old Problem. J. Electrochem. Soc. 2008, 155, A158-A163.

63. Liu, N.; Lu, Z.; Zhao, J.; McDowell, M. T.; Lee, H.-W.; Zhao, W.; Cui, Y., A PomegranateInspired Nanoscale Design for Large-Volume-Change Lithium Battery Anodes. Nat. Nanotechnol. 2014, 9, 187-192.

64. Song, J.; Zhou, M.; Yi, R.; Xu, T.; Gordin, M. L.; Tang, D.; Yu, Z.; Regula, M.; Wang, D., Interpenetrated Gel Polymer Binder for High-Performance Silicon Anodes in Lithium-Ion Batteries. Adv. Funct. Mater. 2014, 24, 5904-5910.

65. Higgins, T. M.; McAteer, D.; Coelho, J. C. M.; Sanchez, B. M.; Gholamvand, Z.; Moriarty, G.; McEvoy, N.; Berner, N. C.; Duesberg, G. S.; Nicolosi, V.; Coleman, J. N., Effect of Percolation on the Capacitance of Supercapacitor Electrodes Prepared from Composites of Manganese Dioxide Nanoplatelets and Carbon Nanotubes. ACS Nano 2014, 8, 9567-9579.

66. McAteer, D.; Gholamvand, Z.; McEvoy, N.; Harvey, A.; O'Malley, E.; Duesberg, G. S.; Coleman, J. N., Thickness Dependence and Percolation Scaling of Hydrogen Production Rate in Mos2 Nanosheet and Nanosheet-Carbon Nanotube Composite Catalytic Electrodes. ACS Nano 2016, 10, $672-$ 683.

67. Chevrier, V. L.; Liu, L.; Le, D. B.; Lund, J.; Molla, B.; Reimer, K.; Krause, L. J.; Jensen, L. D.; Figgemeier, E.; Eberman, K. W., Evaluating Si-Based Materials for Li-Ion Batteries in Commercially Relevant Negative Electrodes. J. Electrochem. Soc. 2014, 161, A783-A791. 

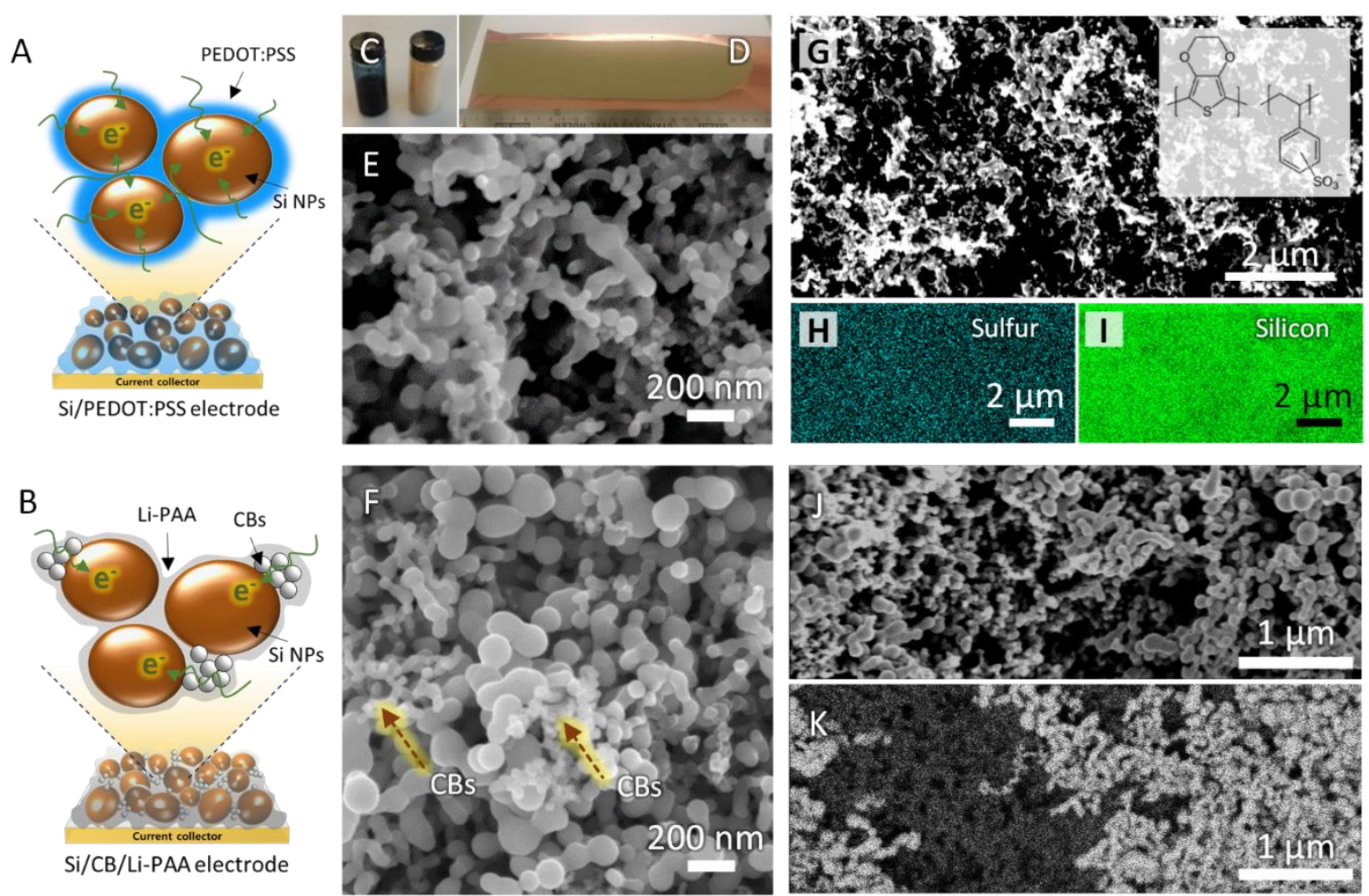

Figure 1. Scheme for the experimental PEDOT:PSS/SiNP electrode (a) and a conventional tricomponent LiPAA/CB/SiNPs electrode (b). The PEDOT:PSS/SiNP composites were produced by mixing the two components (c) to form an aqueous slurry followed by doctor blading onto $\mathrm{Cu}$ foil (d). High magnification SEM images indicate the morphology these two systems (e \& $\mathrm{f}$, respectively). Low magnification SEM image of experimental composite (g) with (h and I) EDS elemental maps of the corresponding area indicating the distribution of sulphur and silicon respectively. The inset in ( $\mathrm{g}$ ) shows the chemical formula PEDOT:PSS. Low magnification SEM image the conventional system (j) with EsB map of corresponding area $(k)$. 

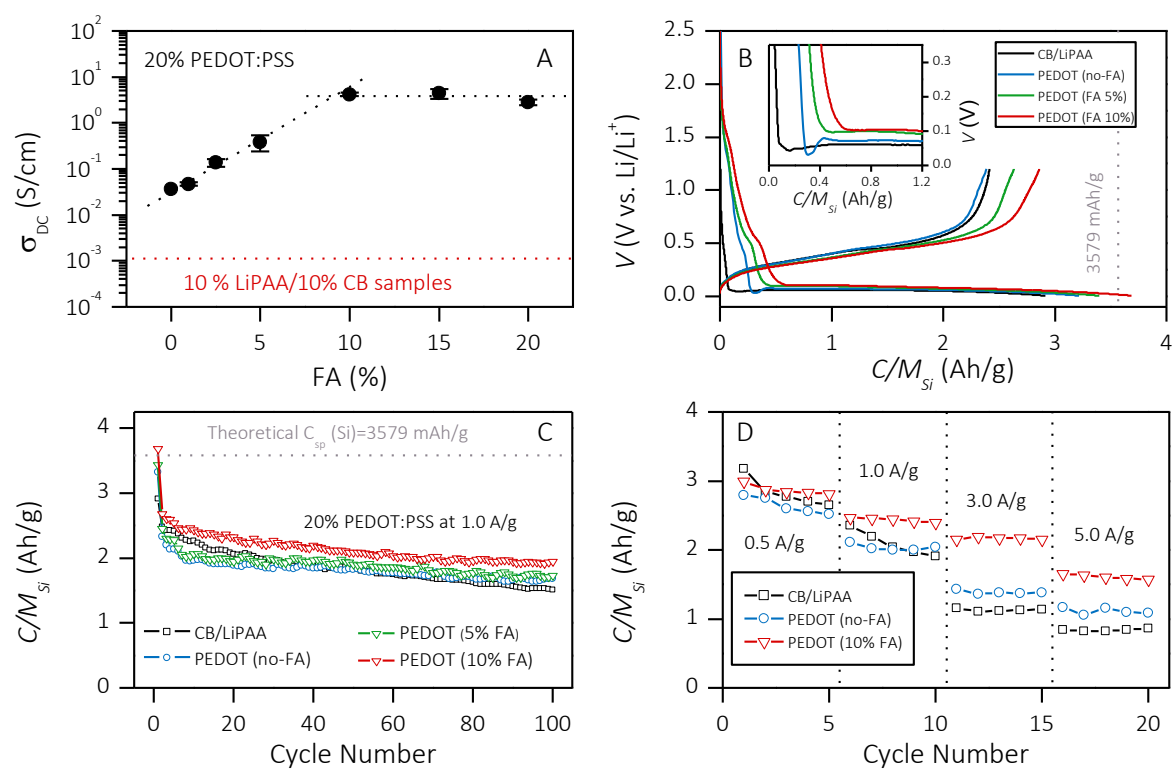

Figure 2. (a) Electrical conductivity for a $20 \mathrm{wt} \%$ PEDOT:PSS/SiNP sample with different amounts of FA secondary dopant added during slurry preparation (expressed as a percentage of the PEDOT:PSS volume). Electrochemical characterisation of CB/Li-PAA/SiNP and PEDOT:PSS/SiNP composites with various FA treatment conditions. (b) Initial lithiation/delithiation profiles at current density of $0.5 \mathrm{~A} / \mathrm{g}$ within 0.005 and $1.2 \mathrm{~V} v s . \mathrm{Li} / \mathrm{Li}^{+}$. The inset shows the enlarged lithiation profiles for all electrodes. (c) Cyclability of lithiation of these electrodes at $1 \mathrm{~A} / \mathrm{g}$ after initial cycling at $0.5 \mathrm{~A} / \mathrm{g}$. (d) Rate capability of these electrodes various current densities from $0.5 \mathrm{~A} / \mathrm{g}$ to $5 \mathrm{~A} / \mathrm{g}$. In all cases, mass loadings of $1 \mathrm{mg} / \mathrm{cm}^{2}$ were used. 


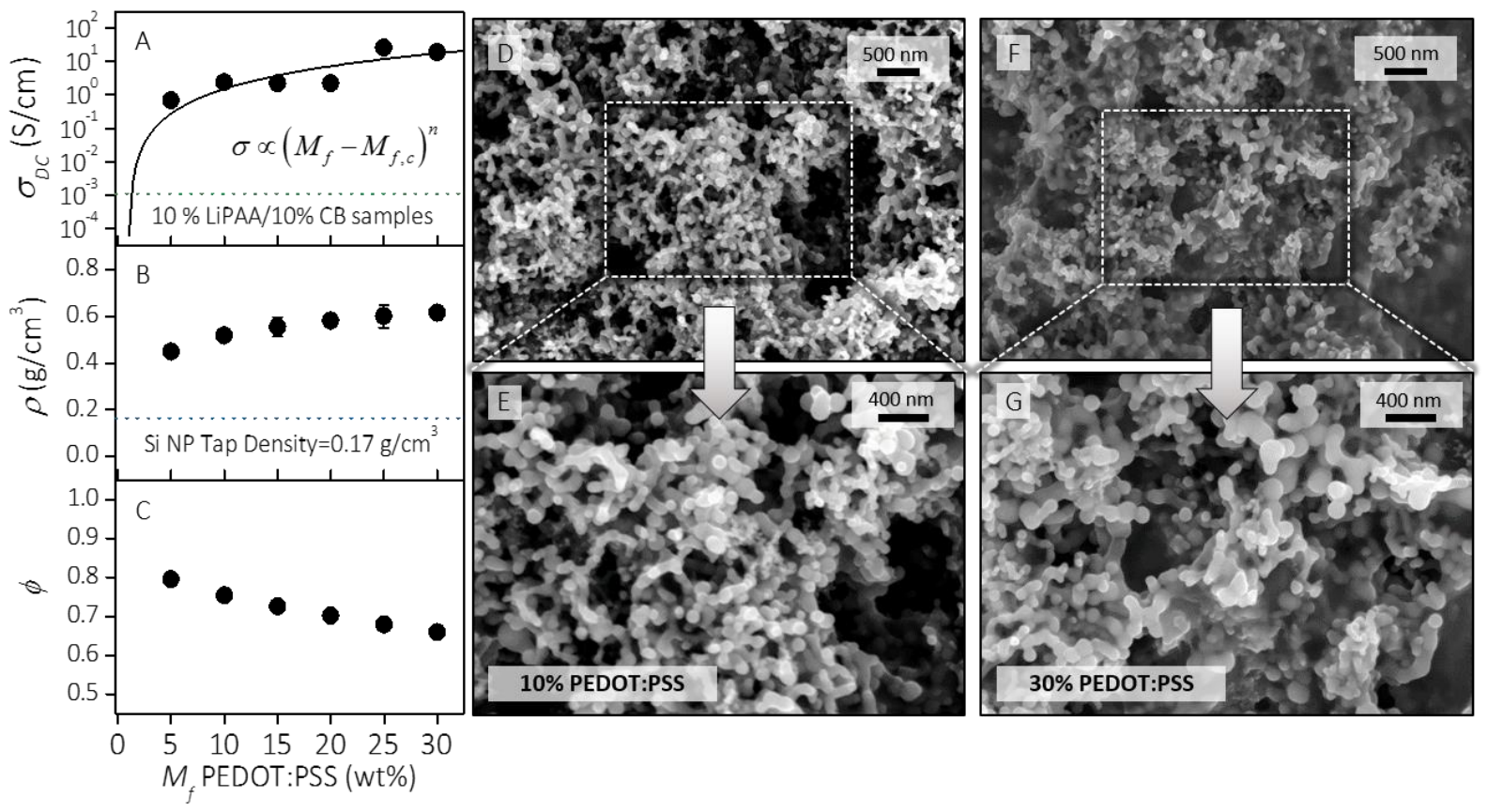

Figure 3. (a) Electrical conductivity (for samples prepared on glass substrates), (b) density and (c) porosity of the PEDOT:PSS/SiNP composites (as-prepared, i.e. uncalendered) as a function of composition. SEM images of a $10 \mathrm{wt} \%$ (d \& e) and $30 \mathrm{wt} \%$ (f \& g) sample at lower and higher magnifications, respectively. 

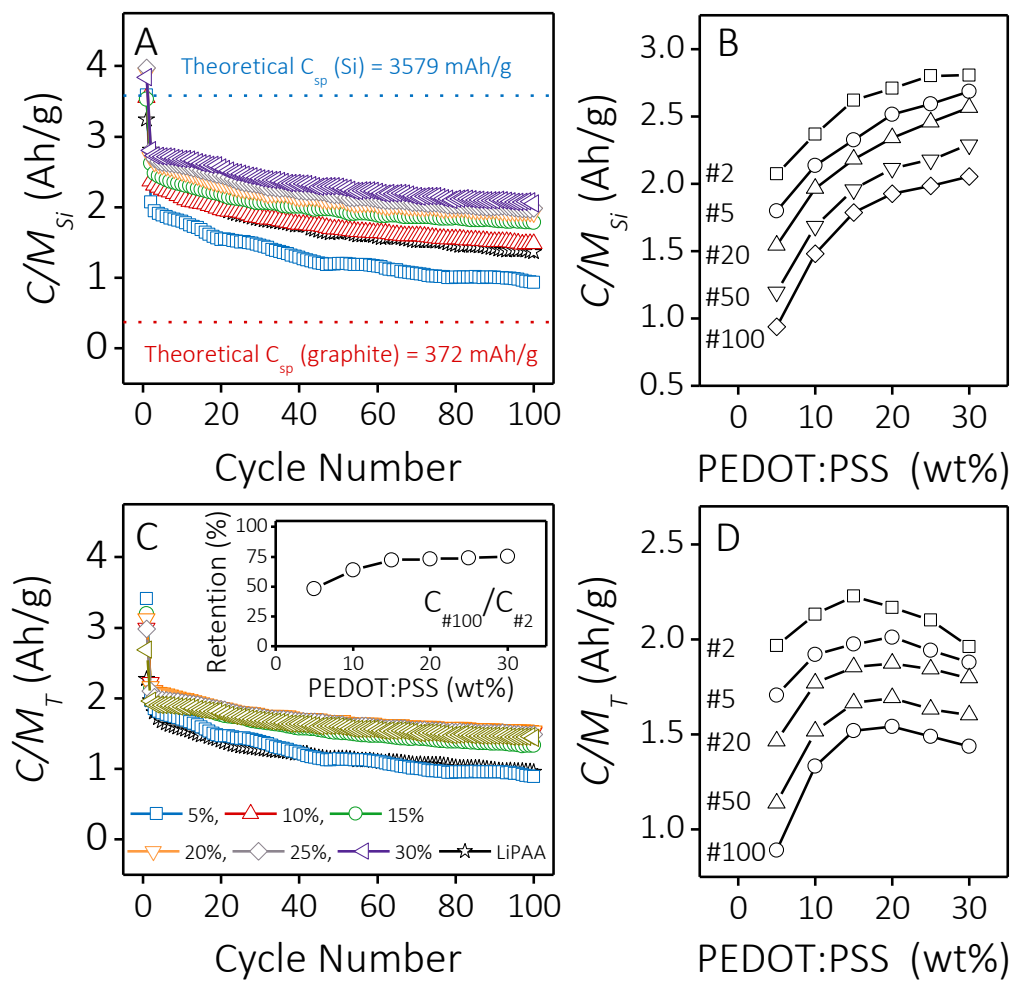

Figure 4. Electrochemical performance of PEDOT:PSS/SiNP electrodes with various compositions. (a) Silicon normalized specific capacity (lithiation) plotted as a function of cycle number. (b) The same values plotted as a function of PEDOT:PSS loading for various cycle numbers. (c) Specific capacity (lithiation) normalized by the total electrode mass $\left(M_{S i}+M_{P E D O T: P S S}\right)$ as a function of PEDOT:PSS loading. (d) The same values plotted as a function of PEDOT:PSS loading for various cycle numbers. The inset in (c) provides the capacity retention ratio at lithiation \#100 relative to lithiation \#2 as a function of PEDOT:PSS loading. 

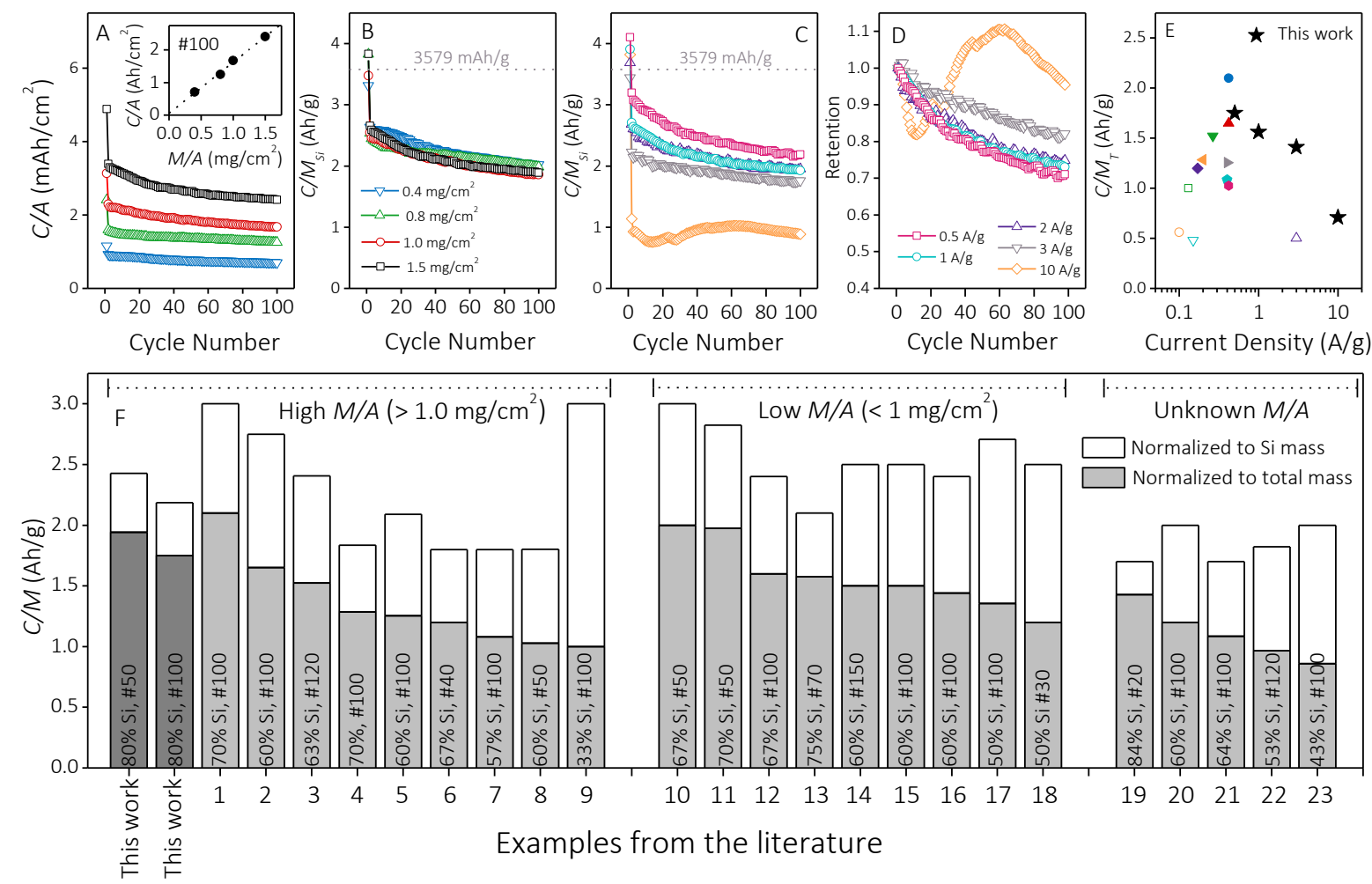

Figure 5. (a) Areal capacity $(C / A)$ and (b) silicon normalized specific capacity $\left(C / M_{S i}\right)$ of a $20 \mathrm{wt} \%$ PEDOT:PSS/SiNP composite with various thickness $\left(0.4\right.$ to $\left.1.5 \mathrm{mg} / \mathrm{cm}^{2}\right)$. The inset in (a) plots the areal capacity vs. mass loading at cycle \#100. (c) Silicon normalized specific capacity and (d) capacity retention (relative to the second cycle) of 20\% PEDOT:PSS composite as a function of cycle number at various current densities $(0.5$ to $10 \mathrm{~A} / \mathrm{g})$. Note, $\mathrm{M} / \mathrm{A}=1.0 \mathrm{mg} / \mathrm{cm}^{2}$ for the electrodes whose data are presented in (c) and (d). (e) Total electrode normalized capacity as a function of current density for a $20 \mathrm{wt} \%$ PEDOT:PSS/SiNP film with $M / A=1.0 \mathrm{mg} / \mathrm{cm}^{2}$ at various current densities. Various literature data are also shown for comparison. A more detailed version of this figure appears is the Supporting Information with a detailed legend. (f) Comparison of the specific capacity of various electrodes when normalized $M_{S i}$ and $M_{\text {total }}$.Values are classified depending on whether the electrode loading is less or greater than $1.0 \mathrm{mg} / \mathrm{cm}^{2}$. Table S5 in the Supporting Information provides details and references. 
ToC fig

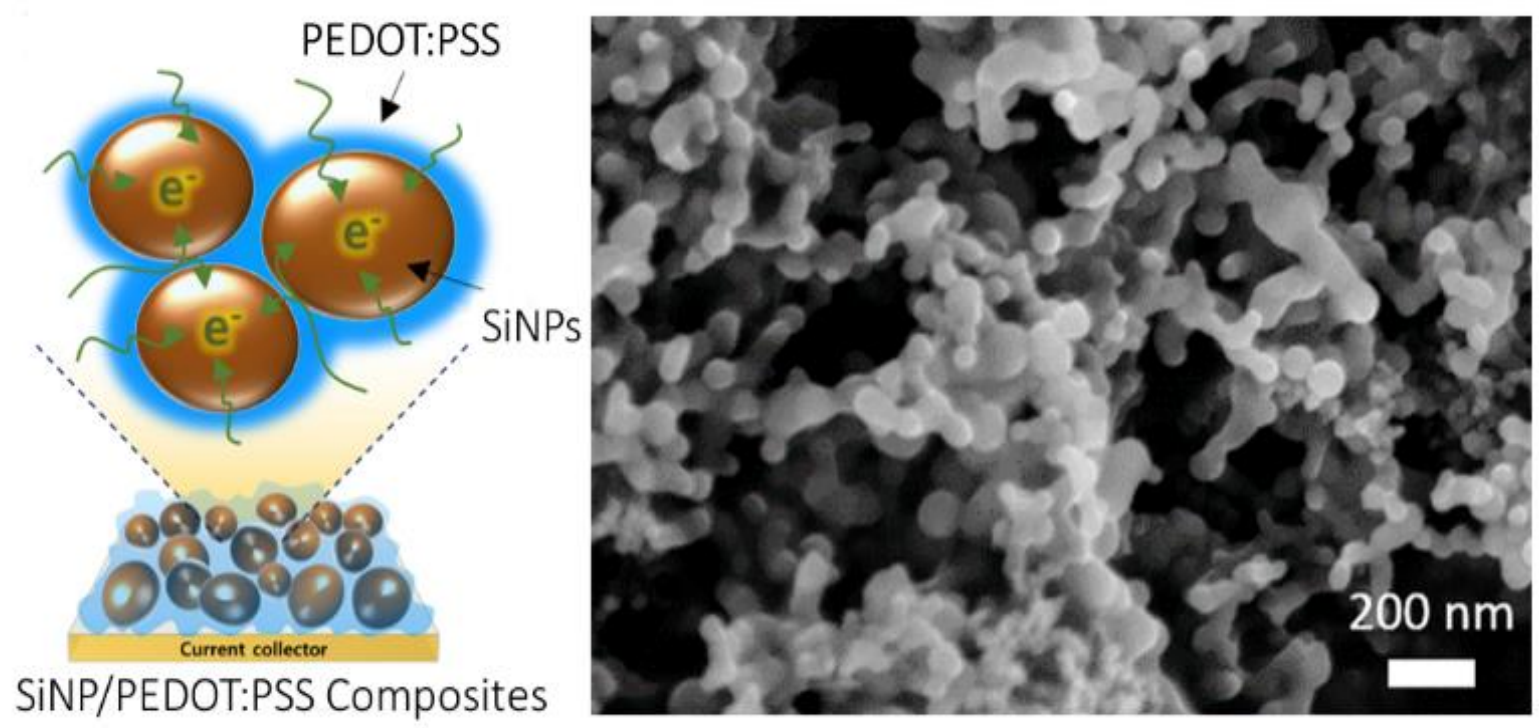

